damage, those consequences will also be permanent. This is faulty logic - on the same basis no one would treat the epilepsy of children with cerebral palsy. Nevertheless, gloomy prognoses can be self fulfilling if they stand in the way of initiating treatment. In many cases, children with hemiplegia and psychiatric problems are more responsive to treatment than children whose psychiatric problems stem from chronic psychosocial adversity. With highly motivated parents, a relatively small amount of informed advice can go a long way. In addition, specific disorders seem likely to respond to the same sorts of specific treatments - such as medication, behavioural therapy, and family therapy-used for other children with comparable psychiatric presentations.

\section{Problems with peer relationships}

Even though most children with hemiplegia are integrated in mainstream schools, problems getting on with other children are common. While most hemiplegic children do have some playmates, they have fewer close reciprocal friendships than most of their classmates, and they are substantially more likely to be teased or bullied. ${ }^{6}$ Prejudice may play a part, but there is increasing evidence for constitutional impairments in social understanding. There is growing interest in children's abilities to understand other people's perspectives, beliefs and intentions; the development of these abilities appears to be delayed in children with hemiplegia, contributing to their emotional and social immaturity.

\section{Practical help}

As a child health professional, what can you do to prevent and treat the psychological complications of your patients with hemiplegia? First, put the issue on the agenda: show through your attitude and questions that you are concerned about psychological as well as physical development. Parents are often greatly reassured to find that their child's problems are common consequences of hemiplegia; the energy previously locked up in self blame can then be diverted into more profitable channels. By telling the family about Hemi-Help, the nationwide parents' support group, you can further reduce the family's sense of isolation and powerlessness by providing them with access to regular newsletters, meetings, and advice (inquiries and help line: 0181-672-3179). Ensuring appropriate educational provision may also depend, at least in part, on health service input. Adequate neuropsychological assessment helps delineate the child's needs and the best ways of meeting those needs; in some areas this assessment is most appropriately carried out by clinical rather than educational psychologists. Reassessment is advisable if a child is running into psychological problems, particularly if the child is becoming hopelessly demoralised by lack of success in all areas of school life. Finally, if the child's psychological problems seem too complicated to assess or treat locally, refer on.

\section{ROBERT GOODMAN}

Department of Child and Adolescent Psychiatry,

Institute of Psychiatry,

De Crespigny Park,

London SE5 $8 A F$

1 Goodman R, Yude C. Do incomplete ascertainment and recruitment matter? Dev Med Child Neurol 1996;38:156-65.

2 Goodman R, Yude C. IQ and its predictors in childhood hemiplegia. Dev Med Child Neurol 1996;38:881-90.

3 Vargha-Khadem F, Isaacs E, Muter V. A review of cognitive outcome after unilateral lesions sustained during childhood. 7 Child Neurol 1994;9:2S672S73.

4 Muter V. Phonology and learning to read in normal and hemiplegic children. (PhD thesis.) London: University of London, 1994.

5 Goodman R, Graham P. Psychiatric problems in children with hemiplegia: cross sectional epidemiological survey. BMF 1996;312:1065-9.

6 Yude C. Peer problems of children with hemiplegia in mainstream schools. (PhD thesis.) London: University of London, 1996

7 Balleny H. Are the concepts of 'theory of mind' and 'executive function' useful in understanding social impairment in children with hemiplegic cerebral palsy. (Clin Psy D thesis.) Norwich: University of East Anglia, 1996.

\title{
Preventative strategies on meningococcal disease
}

The dramatic occurrence of meningococcal disease challenges doctors and attracts intense public interest. It is treatable and preventable, so every death raises the question: Could this have been avoided? Last winter's experience of clusters in schools was the latest round in a struggle to apply rational and effective policies to control disease and panic. A strategist planning to prevent the occurrence and damage of meningococcal disease has various forces to deploy: epidemiology, the recognition of people who are susceptible to infection, vaccines, antibiotics for prophylaxis and treatment, public awareness, and education of doctors and parents. The epidemiology of meningococcal infection is fundamental to any preventive strategy. The incidence in the UK is $2.5-3$ cases/100 000/ year, 1200 to 2000 cases, with exact numbers depending on case definitions and the year in question. ${ }^{12}$ The age specific annual incidence peaks at 6 months of age (50-60/ 100000 ), falls to $2 / 100000$ at age 10 years, rises slightly to $5 / 100000$ in teenagers, and then falls to $1 / 100000$ in adults. $^{3}$

Meningococcal disease illustrates the two purposes of notification: to start public health action and to have statistics on incidence. The consultant in communicable disease control must be notified, to ensure that prophylaxis and advice is given to close contacts and that local general practitioners, schools, and nurseries are informed. A prevention strategy depends on a reliable count of cases. Notifications are an enduring source of statistics, but there has been under-reporting in the past. In the last three years there have been fewer laboratory isolates than notifications, which produced problems in the autumn of 1995 when the public were aware of clusters and an increase in notifications, which was not substantiated by laboratory reports. ${ }^{4}$ The only reliable way to enhance surveillance is to combine sources of data. The laboratory reporting system is accurate for diagnosis, but is an underestimate of incidence since the widespread use of preadmission penicillin, which reduces the proportion of laboratory confirmed cases. Caution in performing lumbar punctures on children may also reduce the numbers confirmed by microbiology. The gap can be reduced by serology, culturing throat swabs from patients on admission and by antigen detection (for example the polymerase chain reaction). ${ }^{5}$ Microscopy of fluid from purpuric lesions is a technique that is sometimes forgotten.

Meningococci are spread by respiratory droplets from the nasopharynx. Disease, if it is going to occur, is within two to 10 days of contact. If illness does not occur, the exposed person who is colonised acquires immunity ${ }^{6}$ but becomes a possible source of infection to others who are exposed to his or her respiratory droplets. 
Meningococci are all members of one species, Neisseria meningitidis, and can be divided into groups according to polysaccharides in the cell wall: serogroups A, B, C, W135, and $\mathrm{Y}$ are well recognised causes of human disease. Group A meningococci are the cause of extensive epidemics in Africa which have spread to the Middle East and the Indian subcontinent. ${ }^{7}$ Group B meningococci are the commonest cause of disease in the Americas, Europe, and Australia; the group is further subdivided by type and subtype antigens. Group C meningococci have caused about a third of the cases in Europe and North America in recent years, including some outbreaks among teenagers and young adults. $^{18}$

\section{Risk factors and special risk groups}

Besides the obvious factors of contact with someone carrying meningococci and a lack of immunity to the strains that are circulating in the community, other risk factors for meningitis are imprecise. Pre-existing respiratory disease, for example influenza, is a factor that could explain the seasonal variation. ${ }^{9}$ Overcrowding has been a factor known since the first world war. Smoking in household members increases the carriage rate $^{10}$ and the risk of disease. ${ }^{11}$ International travel to areas where epidemics are occurring has increased the risk and spread of infection due to group A meningococci. ${ }^{7}$ Smoking and overcrowding are worth prevention strategies in their own right. Hygiene and vaccines have a part in the control of other factors, like influenza $\mathrm{A}$ and travel to epidemic areas.

There are some reasons why a few people have a symptomatic illness and a very few have an overwhelming disease, when most of us have symptomless encounters with meningococci. Being very young (under 1 year) is an obvious factor. A familial deficiency of a terminal component (C7-C9) of complement is rare but multiplies the risk of meningococcal disease by 10000 . Disease is often milder, relapses are more common, and the age of onset is older in this group. Properdin deficiency increases the incidence and mortality of meningococcal disease. ${ }^{12}$ The tumour necrosis factor allele TNF2 has been recently reported as an association with more severe disease. ${ }^{13}$ Asplenism is another suspected reason, although the scale of the risk is uncertain. These people may be more difficult to protect by vaccination, although it should be offered. As with other infectious diseases, herd immunity may be needed to control spread and so protect these susceptible individuals.

\section{Primary prevention}

Primary prevention is aimed at vaccination and the avoidance of the conditions which encourage the spread of meningococci. As the highest age specific incidence is in children under 1 year, vaccination will be most effective when it can be given in infancy, ideally with the other childhood vaccines. Unfortunately there is no antigen common to all serogroups from which a vaccine can be easily derived. Polysaccharide vaccines are available against serogroups A, C, Y, and W135 strains. A single dose of group A polysaccharide vaccine for children over 18 months old, and two doses three months apart for infants, was protective in an epidemic in Finland in 1994-5. ${ }^{14}$ The group C component is only effective in children of 2 years and older, and the resulting immunity lasts three to five years ${ }^{15}$ in children under 4 years old. ${ }^{16}$ To overcome this, the polysaccharides may be conjugated to a protein, for example tetanus toxoid, which converts them to $\mathrm{T}$ cell dependent antigens, thus enhancing immunogenicity in infants and inducing immunological memory. ${ }^{17}$ Conjugated group $\mathrm{C}$ meningococcal vaccines are now undergoing clinical trials in infants. Experience with conjugated
Haemophilus influenzae type b vaccine has shown how successful this strategy might be. If the clinical trials have the expected result of showing that conjugated meningococcal $\mathrm{C}$ vaccine is safe and effective, there will be difficult strategic decisions to make. Assuming that conjugated meningococcal $\mathrm{C}$ vaccine will be included in the routine infant schedule, should there be a large 'catch-up' programme to vaccinate older children, and even university students? Should we ask for a combined conjugated meningococcal $A$ and $C$ vaccine, which would have more international appeal, but perhaps a higher cost? Will there be enough vaccine, the money and the staff available in the school health services and general practices to give the millions of doses that will be demanded? The vaccine that is now available in Britain is a mixture of unconjugated A and C polysaccharides. It is used for travellers abroad to countries where meningococcal A disease is epidemic and occasionally for the control of meningococcal C outbreaks, as described below under secondary prevention.

The development of group B vaccines has been more difficult. ${ }^{18}{ }^{19}$ The capsule of the group B meningococci is poorly immunogenic and the polysaccharide is antigenically similar to a human epitope. Linking the native group B polysaccharide to tetanus toxoid does not improve immunogenicity. A non-capsular vaccine that has shown some promise against group B disease is based on class 1 outer membrane proteins. Moderate efficacy was demonstrated in older children in Cuba, Brazil, and Norway, but once again protection was not given to children under 2 years of age. ${ }^{2021}$ If the development of the Norwegian and other outer membrane protein group B vaccines is successful, the decisions on whom to vaccinate will be as difficult as for the conjugated meningococcal $\mathrm{C}$ vaccine.

\section{Secondary prevention}

The prevention of secondary cases attracts most public interest. As a strategy for prevention, it has serious limitations. As most of the cases are sporadic, secondary prevention can make a relatively small reduction to the incidence and mortality of meningococcal infection. The principle of secondary prevention is based on the observation that a second case occurs within a month in the same household or family of $1 \%$ of primary cases. ${ }^{22}$ The risk is highest in the first week after the first case, but because this risk extends beyond the incubation period, it is thought that secondary cases are often due to transmission within families after the primary case has been admitted to hospital. Treatment of the family is supposed to reduce this risk. It is also recommended that patients with meningococcal disease have chemoprophylaxis before going home. Doctors and the public often misunderstand that chemoprophylaxis is intended to stop spread from carriers, and not to treat early infection. Carriers are likely to be immune, ${ }^{6}$ and the most commonly used antibiotic, rifampicin, is not reliable for treating incubating infections. Penicillin should be used for treating patients with co-primary infection (that it is those with active disease at the time when the index case presents). Such cases are uncommon. The clinical features of early infection maybe unimpressive and vigilance is required. Some have taken this further and recommend a combination of chemoprophylaxis and early treatment for high risk contacts. ${ }^{23}$ There is no good evidence of the effectiveness of this approach, and guidelines should be as simple as possible.

It is clear from the scale of the risk that one would have to do a very large trial to test the efficacy of secondary prevention. It is difficult to see how a controlled trial could now be conducted, but the question may become pressing. Uncontrolled use of antibiotics promotes antibiotic resistance. If rifampicin resistance should increase, following the 
pattern that occurred with sulphonamides, then trials may be necessary to show that drugs that are unlicensed for paediatric use, like ciprofloxacin, have benefits that outweigh the risks. Although the public demand that something should be done is understandable, mass prophylaxis is difficult to do well and increases expectations for similar action on the next occasion. There is substantial doubt about the value of wider use of prophylactic antibiotics. For example, the guidance that nursery school contacts should have chemoprophylaxis has been changed to advice that school and nursery contacts should be considered for prophylaxis only when there are two or more cases (a 'cluster') in a school or similar community within four weeks. If the second case is not the same serogroup or type, or if the diagnosis in one of the cases is unconfirmed, the cases should be treated as sporadic cases and not as a cluster. It must be emphasised that chemoprophylaxis is not foolproof and may fail because meningococci are not eradicated or carriers are not recognised. Failure to prevent secondary cases in families and a nursery have been recorded. ${ }^{24}{ }^{25}$ Therefore chemoprophylaxis must be accompanied by clear advice on the signs of meningococcal disease, preferably using the leaflets produced by the National Meningitis Trust and the Meningitis Research Fund. When a single case occurs in a school or nursery, the other parents should be told about the diagnosis and the signs of meningitis and septicaemia, usually by a letter taken home at the end of school.

The antibiotic usually chosen for chemoprophylaxis is rifampicin, because it clears carriage and because this antibiotic can be given to all ages. ${ }^{26}$ It should be prescribed twice daily for two days in the following doses: for adults and children over 12 years $600 \mathrm{mg}$ (two capsules), for children 1-12 years $10 \mathrm{mg} / \mathrm{kg}$, and for those under 1 year 5 $\mathrm{mg} / \mathrm{kg}$. The paediatric dosage needs to be simpler, because children's weights are often not known in kilograms and fractions of teaspoons or capsules are difficult. Our own regimen is to use rifampicin syrup $(100 \mathrm{mg}$ in $5 \mathrm{ml}$ ) prescribing $50 \mathrm{mg}$ (half a teaspoon) for children under 1 year, $100 \mathrm{mg}$ (one teaspoon) for children age 1-5 years, $200 \mathrm{mg}$ (two teaspoons) for children from 6-10 years, and $300 \mathrm{mg}$ (one capsule or three teaspoons) for children aged $10-12$ years. Another dosage is $3-11$ months $40 \mathrm{mg}(2 \mathrm{ml}$ syrup); $1-5$ years $150 \mathrm{mg}$ ( $7.5 \mathrm{ml}$ of syrup), 6-12 years 300 $\mathrm{mg}$ (one capsule) ${ }^{26}$ Ciprofloxacin for adults and teenagers in a single dose of $500 \mathrm{mg}$ is a useful alternative because it is more available in general practice and because its administration is simpler when large numbers of contacts need to be treated, for example outbreaks in colleges and military camps. Another alternative, which is suitable for pregnant women, is one intramuscular injection of ceftriaxone $250 \mathrm{mg}$ for adults and $125 \mathrm{mg}$ for children less than 12 years. ${ }^{27}$ The doctors attending the patient in hospital can often prescribe for other members of the family; the general practitioner is equally appropriate. In some circumstances the consultant in communicable disease control will prescribe, especially when the close contacts are not in the same family. It is essential that the prescription is given with a clear explanation of its purpose and limitations, that the drug is taken, and that requests from others for prophylaxis are properly addressed.

Vaccination of contacts and groups experiencing outbreaks of meningococcal disease has to be considered if the primary case has group $\mathrm{C}$ or group A disease. Vaccination of close contacts should be preceded by chemoprophylaxis. Vaccination protects the vaccinee within a week of the injection and lasts longer than chemoprophylaxis. It does not promote drug resistance and requires only a single administration. However, vaccination assumes that the index case's serogroup is known, and least protects those most at risk, children under 2 years old. The decision to use meningococcal $\mathrm{A}$ and $\mathrm{C}$ vaccine to contain outbreaks is difficult, more so if there is a lack in confidence in public health services. It is costly to provide in an emergency, and can quickly exhaust the available stocks of $A$ and $C$ vaccine. If it is to be used, it is better given early, for example after the second case in a school or nursery. Further delay, with more cases, may lead to demands for mass vaccination. Such demand may arise in any case. ${ }^{28}$ Clusters usually stop soon after they are recognised and the efficacy of mass vaccination is uncertain, although some persuasive examples are published. ${ }^{29} 30$

\section{Tertiary prevention}

Tertiary prevention refers to the prevention of death or complications in children with clinical infection. It is recommended in Britain that benzylpenicillin (or chloramphenicol if there is an allergy to penicillin) is given as soon as meningococcal infection is suspected, preferably before admission. The National Meningitis Trust and others have campaigned to teach parents and doctors to recognise meningococcal infection early. The emphasis on early diagnosis may heighten public anxiety. Publicity about selected cases of meningitis may imply that some places have a high risk, and miss the point that most cases are sporadic. The leaflets and stories in the press have alerted parents in some cases, but the case fatality rate has been slow to fall from $11 \%$. Publicity can mislead by emphasising meningitis and neglecting the rash (haemorrhagic or maculopapular) of septicaemia. ${ }^{31}{ }^{32}$ Less well described is the mildness of some cases who have symptoms and a rash untreated with antibiotics for several days and still make an uncomplicated recovery. If parents and doctors are to act quickly in order to prevent death and neurological damage, they must recognise that meningococcal disease can start as a mild non-specific febrile illness, without a diagnostic rash or the classical meningeal signs.

\section{Conclusion}

The necessity to ask the question 'Could this be meningococcal disease?' for all patients reveals the limitations of current meningococcal disease control. There is a real prospect of 'bacterial meningitis' vaccines for infants, using conjugate vaccines to cover $H$ influenzae type b, group C meningococci, group A meningococci in epidemic areas, and the commoner types of pneumococci. After these vaccines are introduced, the untidy strategies of secondary prevention will still be needed until there is an effective group B meningococcal vaccine. Only then will we realise the dream of meningococcal disease prevention.

R T MAYON-WHITE P T HEATH

Oxfordshire Department of Public Health,

Oxfordshire Health Authority,

Old Road, Headington,

Oxford OX3 $7 L G$

1 Jones DM, Kazmarski EB. Meningococcal infections in England and Wales: 1994. Commun Dis Rep 1995;5:R125-30

2 Smart LE, Thom L, Reid J, Shearer J, Girdwood RW. Meningococcal disease in Scotland 1994. Communicable Disease and Enviromental Health in Scotland Weekly Report 1995;29:66-9.

3 Jones DM, Mallard RH. Age specific incidence of meningococcal infection England and Wales 1984-1991. F Infect 1993;27:83-8.

Anonynous. Update on invasive meningococcal infections. Commun Dis Rep 1995;5(47):255

5 Kaczmarski EB, Cartwright KA. Control of meningococcal disease: guidance for microbiologists. Commun Dis Rep 1995;5:R196-8.

6 Goldsteiner I, Gotschlich EC, Artenstein MS. Human immunity to the meningococcus: II. Development of natural immunity. $\mathcal{f}$ Exp Med 1969;129:1327-47.

7 Moore PS, Reeves MW, Schwartz B, Gellin BG, Broome CV. Intercontinental spread of an epidemic group A Neisseria meningitidis strain. Lancet nental spread of an epidemic group A Neisseria meningitidis strain. Lance

8 Jackson LA, Schudat A, Reeves MW, Wenger JD. Serogroup C meningococcal outbreaks in the USA: an emerging threat. $\mathscr{f} A M A 1995 ; 273: 383-9$. 
9 Cartwright KA, Jones DM, Smith AJ, Stuart JM, Kaczmarski EB, Palmer SR. Infuenza A and meningococcal disease. Lancet 1991;338:554-7.

10 Stuart JM, Cartwright KA, Robinson PM, Noah ND. Effect of smoking on meningococcal carriage. Lancet 1989;ii:723-5.

1 Stuart JM, Cartwright KA, Dawson JA, Rickard J, Noah ND. Risk factors for meningococcal disease. Community Medicine 1988;10:139-46.

12 Cuncliffe NA, Snowden N, Dunbar EM, Haeney M. Recurrent meningococcal septicaemia and properdin deficiency. F Infect 1995;31:67-8.

13 Nadel S, Newport MJ, Booy R, Levin M. Variation in the tumour necrosis factor- $\alpha$ gene promoter region may be associated with death from meningococcal disease. F Infect Dis 1996;174:878-80.

14 Peltola A, Makela PH, Kayhty H, et al. Clinical efficacy of meningococcus group A capsular polysaccharide vaccine in children three to five years of age. $N$ Engl f Med 1977;297:686-91

15 Lepow ML, Goldsteiner I, Gold R, Randolph M, Gotschlich EC. Persistance of antibodies following immunization of children with groups A and C meningococcal polysaccharide vaccine. Pediatrics 1977;60:673-80.

16 Reingold AL, Broome C, Hightower AW, et al. Age specific differences in duration of clinical protection after vaccination with meningococcal duration of clinical protection after vaccination

17 Twumasi PA, Kumah S, Leach A, et al. A trial of a group A plus group C meningococcal poly-saccharide-protein conjugate in African infants. $\mathcal{F}$ meningococcal poly-sacchari

18 Ala'Aldeen DAA, Cartwright KAV. Neisseria menigitidis: vaccines and vacAla'Aldeen DAA, Cartwright KAV. Neiss
cine candidates. F Infect 1996;33:153-7.

19 Herbert M, Heath PT, Mayon-White RT. Meningococcal vaccines in the United Kingdom. Commun Dis Rep 1995;5:R130-5.

20 Bjune G, Hoiby EA, Gronnesby JK, et al. Effect of outer membrane vesicle vaccine against group B meningococcal disease in Norway. Lancet 1991;338:1093-6.

21 de Moraes JC, Perkins BA, Camargo MC, et al. Protective efficacy of a serogroup meningococcal vaccine in Sao Paulo, Brazil. Lancet 1992;340:1074-8.
22 Cooke RPD, Riordan T, Jones DM, Painter MJ. Secondary cases of meningococcal infection among close family and household contacts in England and Wales, 1984-7. BMF 1989;298:555-8.

23 Kristiansen BE, Knapskog AB. Secondary prevention of meningococcal disease. BM7 1996;312:591-2

24 Foster G, Panigrahi H, Walker M. Failure of chemoprophylaxis to prevent meningococcal disease. BMF 1986;292:886-7.

25 Cartwright KA, Hunt D, Fox A. Chemoprophylaxis fails to prevent a second case of meningococcal disease in a day nursery. Commun Dis Rep 1995;5:R199.

26 PHLS Meningococcal Infections Working Party on and Public Health Medicine Enviromental Group. Control of meningococcal disease: guidance for consultants in Communicable disease control. Commun Dis Rep 1995;5:R189-95.

27 Scwartz B, Al-Tobaiqi, Al-Ruwais A, et al. Comparitive efficacy of ceftriaxone and rifampicin in eradicating pharyngeal carriage of group A Neisseria meningitidis. Lancet 1988;i:1239-42.

28 Hume SE. Mass voluntary immunization campaigns for meningococcal disease in Canada: media hysteria. $7 A M A$ 1992;267:1833-8.

29 Masterton RG, Youngs ER, Wardle JC, Croft KF, Jones DM. Control of an outbreak of group $\mathrm{C}$ meningococcal meningitis with a polysaccharide vaccine. F Infect 1988;17:177-82.

30 Pearce MC, Sheridan JW, Jones DM, et al. Control of group C meningococcal disease in Australian aboriginal children by mass rifampicin chemoprophhylaxis and vaccination. Lancet 1995;346:20-3.

31 Thomson AP, Hayhurst GK. Press publicity in meningococcal disease. Arch Dis Child 1993;69:166-9.

32 Riordan FAI, Thomson APJ, Sills JA, Hart CA. Who spots the spots? Diagnosis and treatment of early meningococcal disease in children. BMF 1996; 313:1255-6 